\title{
Design of Commercial Vehicle Information Collection and Remote Monitoring System
}

\author{
Yi ZHANG ${ }^{a}$, Yong $X U^{b}$, \\ School of Electronic Engineering and Automation, \\ Guilin University of Electronic Technology, Guilin 541004, China

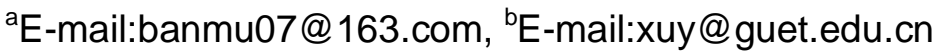

\begin{abstract}
Keywords: Vehicle System; ARM; CAN Bus; SAE J1939 Protocol; Remote Monitoring;
Abstract. Traditional commercial automobile vehicle terminals provide insufficient display of information and make it difficult to extract automobile data. To address these problems, a commercial automobile information system is designed which includes S5PV210 as its main controller. The system acquires automobile data via the CAN-bus and performs analysis per SAE J1939 protocol, displays in real time the information on the vehicle terminal and, by use of a $3 \mathrm{G}$ network, enables real-time monitoring in the remote monitoring system. The design incorporates an expansion card which uses STM32 as the controller and, by means of acceleration sensing and RFID technologies, achieves driver identification and automatic accident reporting. The entire system comes with simple and tidy interfaces and easily expandable hardware and software, able to satisfy the diversified needs.
\end{abstract}

\section{Introduction}

Along with the incessant progress in automobile electronic technologies, on-board sensors and electronic control units (ECUs) grow in large quantities, sending automobiles onto an intelligent development road. CAN-bus has been applied extensively to automobiles to complete data exchange between vehicle ECUs and sensors. The vehicle information terminal, as an important node in the CAN network, processes, displays, and stores automobile data [1,2].

For the mainstay vehicle terminals available in China, their panels provide only a small amount of basic automobile information. The vehicle screens are most used for navigation and entertainment, so the large quantity of data generated by automobile sensors and ECUs can only be interpreted and analyzed with the aid of an external specialty instrument, not possible for real-time display on the on-board terminal, let alone remote data monitoring. Moreover, the closeness of traditional on-board terminals makes it difficult to carry out personalized secondary development.

To cope with such problems associated with commercial automobile on-board terminals, an open system platform is designed on the basis of ARM processor and Linux OS. This system offers good interaction experience, satisfies diversified needs for vehicle information system, and enables in-time processing, display, and remote monitoring of automobile data. It breaks the closeness of its predecessors by allowing customized installation of modules to suit individual needs and by expanding the system's application and functionality.

\section{Function Design}

The vehicle information system hardware is composed of two major components: vehicle information terminal and extension card. Powered by a Cortex-A8 processor, the vehicle information terminal not only fulfils the functions such as navigation, multimedia entertainment, and 3G data connection but also analyzes the CAN messages per SAE J1939 protocol, stores them, and uploads them to the server [3]. The expansion card is designed for commercial automobiles often traveling in sparsely populated remote regions for an extended time, during which the driver may get tired after a long time of driving and an accident might be reported not in good time. Considering such a scenario, the card, equipped as the core with an STM32 chip, performs such functions as driver identification, warning against long-time or tired driving, and automatic accident reporting. The user may select different expansion cards to suit their needs, adding ample expandability to the vehicle system. 


\section{Design of Hardware}

Design of Controller Hardware. The vehicle information terminal puts forward higher requirements on the main controller in order to achieve operating system running smoothly, vehicle information collection, human-computer interaction etc. The S5PV210 which is a 32-bit RISC microprocessor launched by Samsung is used as the main controller of the terminal.S5PV210 integrates the ARM Cortex-A8 core, which implements the ARM architecture V7-A with supporting peripherals. S5PV210 adopts 64-bit internal bus architecture, and includes many powerful hardware accelerators for tasks such as motion video processing, display control, and scaling. The core-board extends 1GB DDR2 SDRAM memory and 1 GB Nand flash memory. According to the above, the embedded Linux system can run smoothly.

The expansion card is equipped with a STM32F4 series single chip processor as the controller launched by ST. It has rich peripherals, cost-effective and low power consumption. The chip connects an acceleration sensor measuring the acceleration of the vehicle via the $\mathrm{I}^{2} \mathrm{C}$ interface and a RFID card reader via the SPI interface. The system hardware architecture is shown in Fig. 1.

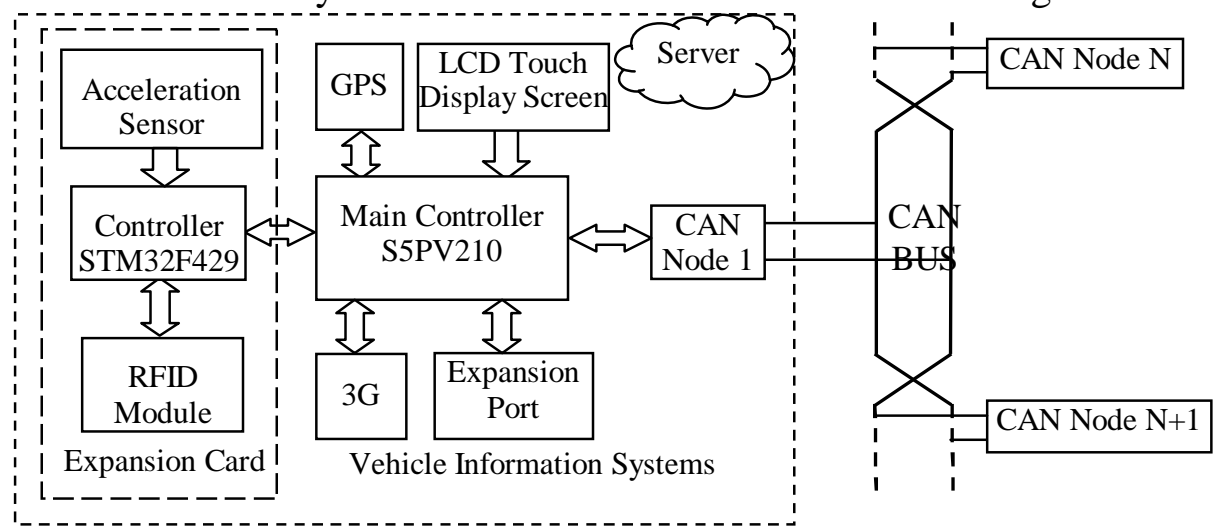

Fig. 1 The system hardware architecture

CAN communication Module. The MCP2510 is a full CAN protocol controller implementing. It supports CAN 2.0A/B versions of the protocol, and is capable of transmitting and receiving standard and extended messages. It is also capable of both acceptance filtering and message management. It includes three transmit buffers and two receive buffers that reduce the amount of microcontroller management required. The MCU communication is implemented via SPI with data rates up to 5 $\mathrm{Mb} / \mathrm{s}$.

CAN driver, with chip PCA82C250 as the core, has excellent electromagnetic compatibility.The PCA82C250 is the interface between the CAN protocol controller and the physical bus. The device provides differential transmit capability to the bus and differential receive capability to the CAN controller.

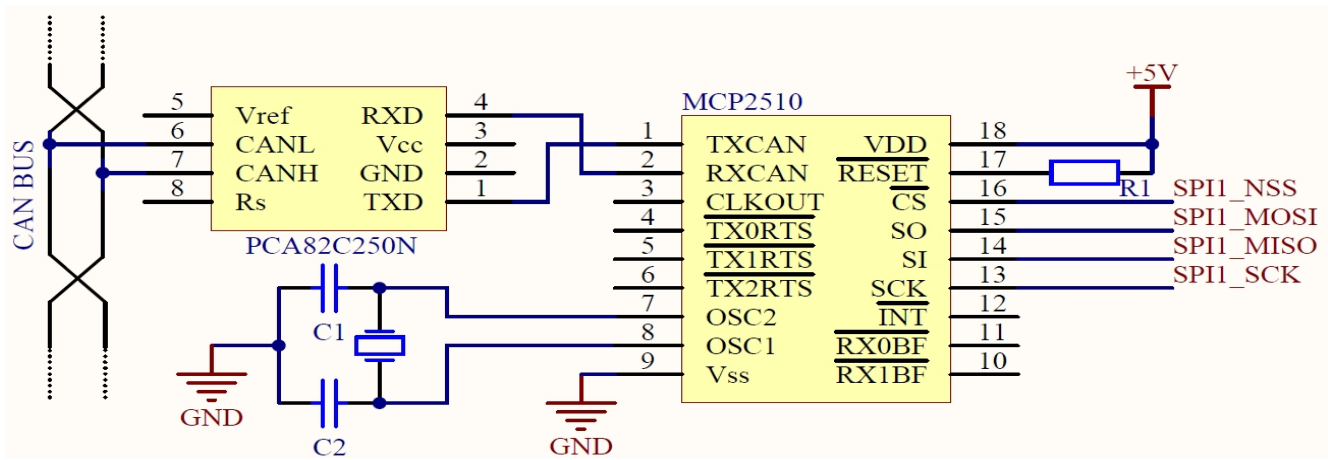

Fig. 2 MCP2510 peripheral circuit

RFID Card Reader. The MFRC522 is a highly integrated reader/writer for contactless communication at $13.56 \mathrm{MHz}$.It supports ISO 14443A mode. The MFRC522's internal transmitter part is able to drive a reader/writer antenna designed to communicate with ISO 14443A cards and transponders without additional active circuitry [4]. 
Acceleration Sensor. The ADXL345 is a small, thin, ultralow power, 3-axis accelerometer with high resolution (13-bit) measurement at up to $\pm 16 \mathrm{~g}$. Digital output data is formatted as 16-bit twos complement and is accessible through either a SPI or $\mathrm{I}^{2} \mathrm{C}$ digital interface.The ADXL345 is well suited for extended temperature range industrial and aerospace equipment[5]. It measures the static acceleration of gravity in tilt-sensing applications, as well as dynamic acceleration resulting from motion or shock.

\section{Software Design}

The Software Design of System. With the VMware Workstation10.0 and Ubuntu14.06 development environment on PC platforms, this system transplants the embedded Linux system and Qt graphical user interface application on the ARM platform for development framework, and then uses Qt Creator4.0 for UI interface design. Qt provides an efficient and flexible development framework, realizing the communication between objects through signals and slots mechanism. When the vehicle terminal is electrified, the system will boot the u-boot program to run and call the Linux kernel to start, and once started successfully, the operating system will automatically call Qt .

The CAN Message Parsing. SAE J1939 is a vehicle multilayer communication protocol based on CAN2.0B standard developed by the American society of automotive engineers (SAE) to support the vehicle communication between the CAN nodes. Be set to the rate as $250 \mathrm{Kbps}$ the protocol has been widely used in commercial vehicles. It defines the data link layer, network layer, application layer and network management based on CAN2.0B in order to develop the superior performance of CAN network[6,7].

The data link layer defines the data structure and encoding rules of the information frame, including communications, transport, communications, bus arbitration priority, etc. It regroups and redefines the 29 bit identifier of the CAN extended frame. In this situation the identifier can describe all the features of packet .J1939 frame and CAN extended frame format cross-references show in Table 1.

Table 1. J1939 frame and CAN extended frame format cross-references

\begin{tabular}{|c|c|c|c|c|c|c|c|c|c|c|c|c|c|c|c|}
\hline Extended & SOF & \multicolumn{4}{|c|}{ Identifier } & SRR & IDE & \multicolumn{3}{|c|}{ Extended } & RTR & R1 & R0 & DLC & Data \\
\hline J1939 & SOF & P & R & DP & PF & SRR & IDE & PF & PS & SA & RTR & R1 & R0 & DLC & Data \\
\hline Length & 1 & 3 & 1 & 1 & 6 & 1 & 1 & 2 & 8 & 8 & 1 & 1 & 1 & 4 & $0-64$ \\
\hline
\end{tabular}

Various parameters about the vehicle control and communication defined by SAE J1939 protocol in the application layer including the suspicious parameter number (SPN) and the parameter group number (PGN).Commonly parameter information shows in Table 2.

Table 2. Commonly parameter information

\begin{tabular}{|c|c|c|c|c|c|c|}
\hline SPN & Parameter Name & Length & Resolution & Unit & PGN & Parameter Group Name \\
\hline 190 & Engine speed & 2 Byte & 0.125 & $\mathrm{rpm}$ & 61444 & Electronic engine control \\
\hline 84 & Wheel speed & 1 Byte & $1 / 256$ & $\mathrm{~km} / \mathrm{h}$ & 65265 & Vehicle Speed \\
\hline 185 & Average fuel economy & 2 Byte & $1 / 512$ & $\mathrm{~km} / \mathrm{kg}$ & 65266 & Average economy \\
\hline 110 & Engine Coolant temperature & 1 Byte & 1 & ${ }^{\circ} \mathrm{C}$ & 65262 & Engine temperature \\
\hline 100 & Engine lube oil pressure & 1 Byte & 4 & $\mathrm{kpa}$ & 65263 & Engine liquid level \\
\hline
\end{tabular}

Obtain the value of PGN, according to Table 1 J1939 frames and CAN extend frame corresponding relation, when receive the CAN extend frame packets through the network CAN. PGN $=\mathrm{R}+\mathrm{DP}+\mathrm{PF}+\mathrm{PS}, \mathrm{R}$ for retain, $\mathrm{DP}$ for data pages, $\mathrm{PF}$ for the protocol data unit format, PS for PDU Specific. After getting PGN values, calculate the physical values by the parameter and resolution in table 2 .

Software Design of Expansion Card. When selecting open extension function, expansion card powers on. STM32 is configured the SPI interface to realize the communication with RFID card reader module and the $\mathrm{I}^{2} \mathrm{C}$ interface to realize the communication with ADXL345 acceleration sensor module. Processes shows in Fig. 3. 


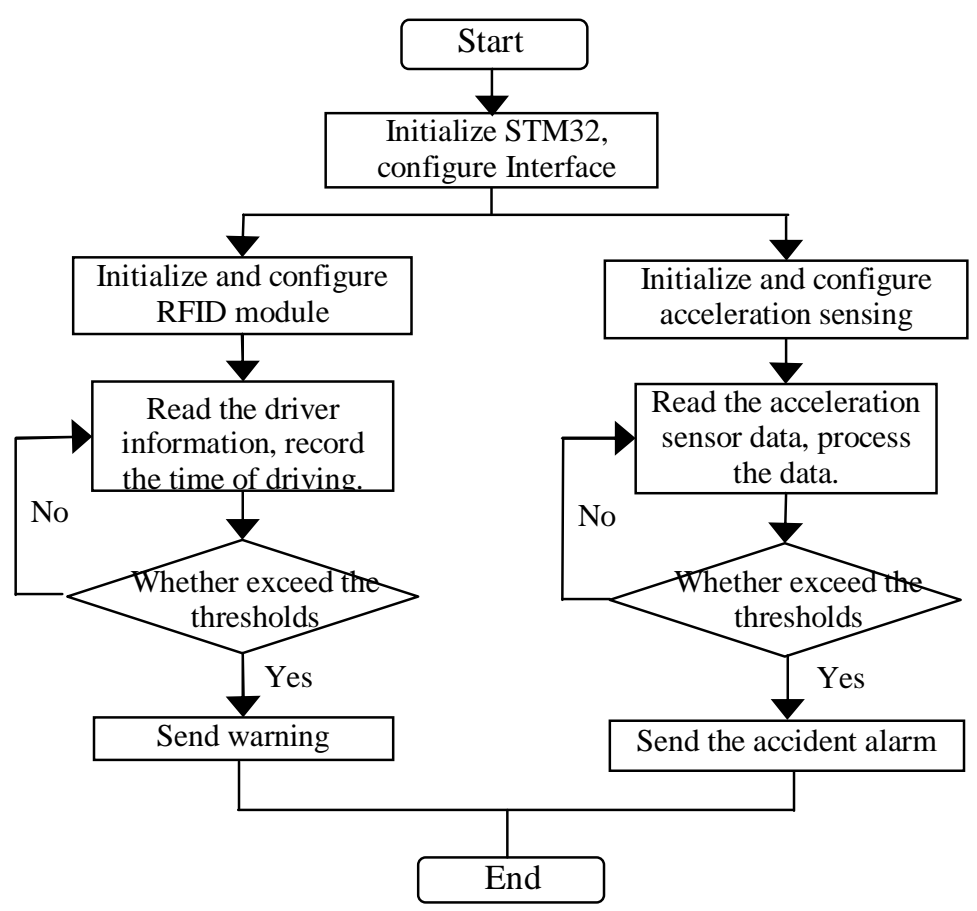

Fig. 3 Expansion card program flow chart

After the ADXL345 is electrified, 1.1ms delay is needed, and then the register shall be initialized, with the range of $16 \mathrm{~g}, 13$-bit full resolution output and the output data rate of $200 \mathrm{~Hz}$. In order to reduce the influence of vehicle vibration on test results, five consecutive measurements are averaged and compared to threshold values. When the detected acceleration data exceeds the set threshold values, the collision accident is deemed to be occurred. The interrupt signal will be triggered, and then the vehicle terminal after receiving the interrupt signal will send alarm signal to notify the remote monitoring system through $3 \mathrm{G}$ network.

The RFID card reader module achieves driver identification by reading the driver's smart card ID number. When the module is running, it judges whether within the scope of the antenna having a smart card repeatedly. When it finds a smart card, the RFID card reader reads smart card ID number after preventing collision process and verification. The driver information will be found in the database according to the ID number, and shows in vehicle terminal.

Software Design of Remote Monitoring System. Software on the remote server side chooses LabVIEW as a development tool, and adopts the graphical programming language to write programs that can be used to easily create a user interface. In the interface, the static map API is implanted in Baidu map, which can directly monitor the location of vehicles. Service address: http://api.map.baidu.com/staticimage/v2[8]. Vehicle remote monitoring system interface showns in Fig.4.

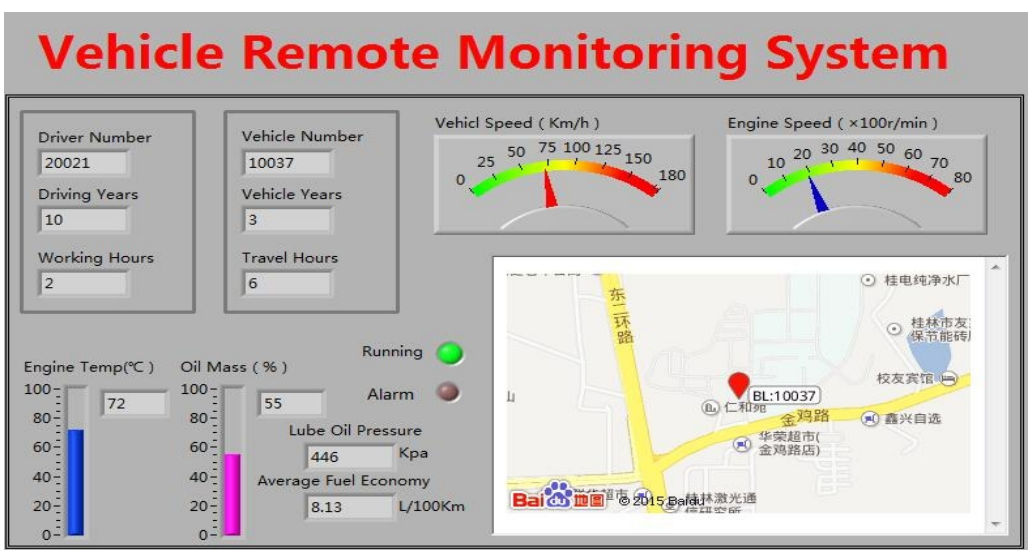

Fig.4 Vehicle remote monitoring system interface 


\section{System Function Testing}

CAN bus system uses the simulated vehicle CAN network built by CAN Analyzer CANalyst-II for test. CAN Analyzer sets the frame ID and data domain for a variety of CAN messages based on SAE J1939 protocol, and then sends them with the circle of $500 \mathrm{~ms}$ to simulate the CAN nodes of commercial vehicles and send vehicle information,like engine speed,average fuel economy. Through the simulated vehicle body CAN bus network, the CAN nodes on vehicle terminals successfully read CAN messages and parse vehicle information based on SAE J1939 protocol that can be displayed on vehicle terminals, and the dynamic data of vehicles can be successfully received through $3 \mathrm{G}$ network in remote monitoring system.

A test platform has been set up to test the automatic accident reporting function by freescale smart car model. Due to the car model is small, the expansion card fixed on it is tested as a separate module. The car model's battery supplies the expansion card power by converting battery voltage to $3.3 \mathrm{~V}$ by conversion chip. When there is alarm, LED flash. In order to reduce destruction of the experiment, the automatic reporting acceleration threshold is set to $1.5 \mathrm{~g}$.

\section{Conclusion}

Based on ARM processor, the vehicle information system respectively designs the vehicle terminal and expansion card. Taking SAE J1939 protocol as the core, the system allows the vehicle terminal and remote monitoring system both can instantly get the vehicle information through the real-time processing and analysis of CAN network node data on vehicle terminals; and the driver identification, automatic accident alarm and other functions are provided for commercial vehicles; through the design of hardware and software, the general functions and individual needs of vehicle information system are realized in modules, which has rich diversity and expansibility and provides a new solution for commercial vehicle information system.

\section{Acknowledgements}

This work was financially supported by the key Laboratory of Guangxi Automatic Detection Technology and Instrument Foundation (YQ14111).

\section{References}

[1] S.Jankovic, D.Kleut and I.Blagojevic: IEEE 9th International Symposium on Intelligent Systems and Informatics (Subotica 2011).

[2] G. Cena, I.C. Bertolotti and T.Hu: IEEE Transactions on Industrial Informatics. Vol. 9(2013), p. 2155

[3] G.S. Zhang, S.J. Wang: Automotive Engineering. Vol. 38(2015), p.980.

[4] E. Imran and A. Emin: Wireless Personal Communications. Vol. 59(2011), p.43.

[5] B. Subhamoy, M. Krishna and L. Domenico: Soil Dynamics and Earthquake Engineering. Vol.36 (2012), p.111.

[6] Y.L.Hu: Based on the J1939 protocol implement information collection system of vehicle terminal (MS., Wuhan University of Technology, China 2012), p.14.

[7] S.M. Marx, J.D. Luck and R.M. Hoy: Computers and Electronics in Agriculture. vol.118 (2015), p.179.

[8] Information on http://developer.baidu.com/map/index.php?title=static. 